\title{
Experimental detection of local instability in materials
}

\author{
M. Emami Tabrizi ${ }^{1, a}$, B. Halphen ${ }^{1}$, M.P. Luong ${ }^{1}$ and J.C. Eytard ${ }^{1}$ \\ ${ }^{1}$ LMS, Solids Mechanics Lab., Civil Eng., Ecole Polytechnique, 91128 Palaiseau Cedex, Fr.
}

\section{Introduction}

This paper introduces a nondestructive technique based on ultrasound propagation characteristics to detect material instability occurred in geomaterials such as brittle rocks and concrete. A special testing technique was used to detect the occurrence of nonlinear characteristics of ultrasound propagation. An input-output non-parametric procedure based on wave propagation and a nonlinear analyzer for data reduction procedure were chosen to portray the unstable behavior of a specimen subjected to increasing static compressive loads. Based on a multidimensional Fourier transform, the nonlinear analyzer permits to separate linear and nonlinear parts and to monitor nondestructively and continuously the overall alteration or damage process of brittle material so that damage mechanisms could be quantitatively estimated by a dimensionless parameter the so-called nonlinearity ratio.

\section{Proposed technique}

Techniques using ultrasonic waves are especially appealing because of the direct connection between the characteristics of the wave propagation and the damage states of a solid [1]. The present analysis only considers, in the framework of small perturbations, the propagation mechanism of a small wave through an elastic plastic solid presenting locally an unstable behavior. Within the theory of plasticity, several postulates that guarantee the mechanical stability of frictional materials have been suggested based on either energy or wave propagation considerations. For a non-associated plastic flow rule, localization can appear during hardening. This type of instability, referred to as pre-failure flow instability $[2,3]$. Based on the assumption that a stable material is able to propagate a small perturbation in the form of wave, it can be showed that a wave propagates in a material with an elastic plastic matrix A, along the direction $\alpha$, if and only if all the eigenvalues $\lambda$ of the matrix B of propagation are real and positive. This phenomenon is used as a highly sensitive manifestation of geomaterial instability $[3,4]$. The proposed method aims to evaluate the significance of non-linear terms in the global response [3]. If these nonlinear aspects exhibit a sudden increase, it will be associated to the occurrence of material instability causing additional non-linear behavior. To quantify the non-linear contributions in the global response, ${ }_{l} r_{m}$ the nonlinearity ratio at point $l$ for the $m^{\text {th }}$ excitation is defined as follows:

$$
r_{m}=\frac{\sum_{i=2}^{N}, E_{m}^{i}}{\sum_{i=1}^{N} E_{m}^{i}}=\frac{\sum_{i=2}^{N} B^{2 i} \eta_{i}}{\sum_{i=k}^{N} B^{2 i} \eta_{i}}=\frac{\text { non }- \text { linear energy }}{\text { total energy }} \quad \eta_{i}=\int_{0}^{\infty} k_{i}(t, t, \ldots, t)^{2} d t
$$

where $k_{i}$ : pseudo kernels, $t$ : time, $E_{m}^{i}$ : energy of the $i^{\text {th }}$ component of dynamic response for the $m^{\text {th }}$ excitation.

a e-mail : emami@1ms.polytechnique.fr

This is an Open Access article distributed under the terms of the Creative Commons Attribution-Noncommercial License 3.0, which permits unrestricted use, distribution, and reproduction in any noncommercial medium, provided the original work is properly cited. 


\section{Experimental validation}

The proposed technique has been applied in laboratory on the different specimens subjected to increasing levels of static compressive loading. The pulse transmission technique has been applied as a nondestructive testing. The pulser section produces an electrical pulse to excite a piezoelectric transducer, which emits an ultrasonic pulse. The experimental set-up continuously monitored the applied load, the axial and radial strains and the signals of ultrasonic waves "Fig. 1".

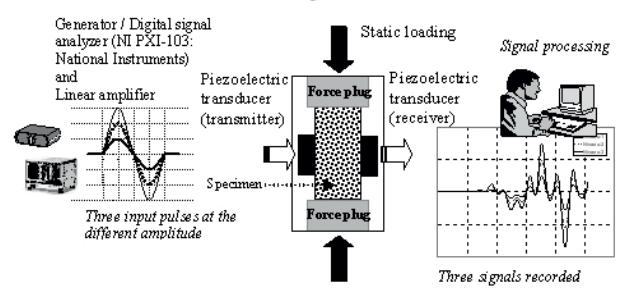

Fig. 1. Experimental device of the ultrasound propagation and signal processing.

The data processing technique provides the variation of nonlinear energy normalized by the total energy and nonlinearity contrast as a function of stress levels "Fig. 2a". They have evidenced a material stability threshold "ST" that precedes significantly the occurrence of crack initiation so that either load-controlled or displacement controlled tests can be used. The acoustic emission AE is represented by points in "Fig. 2b". The results also show that the lack of significant AE activity in the initial stages of loading makes it more difficult to detect the occurrence of crack initiation (ST). However, the unstable crack threshold could be readily determined during the compression loading.

(2a)

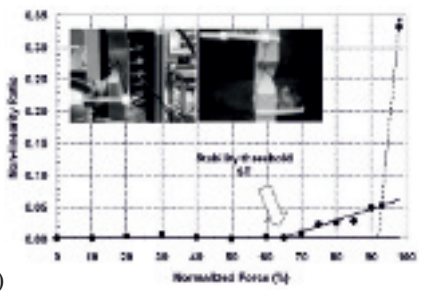

(2b)

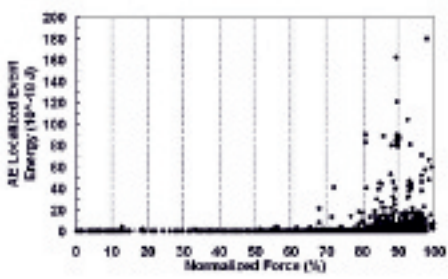

Fig. 2. Graphical definition of the stability threshold ST (2a) \& Distribution of localized AE events (2b).

\section{Conclusion}

This nondestructive evaluation of material instability could offer an efficient field monitoring before failure initiation in order to reduce risk of imminent failures by giving advanced and sufficient warning for remedial measures to be designed. The application of ultrasound scanning to inspection and monitoring of structures relies on the fact that during the process of micro-cracking, the geomaterial may be locally unstable and hence modifies and partially obstructs the propagation characteristics of ultrasound pulses. One of the advantages of using NL monitoring technique is the possibility to observe damage processes during the entire load history without any disturbance to the structures. Measurement can be performed from remote locations, adding another distinct advantage.

\section{References}

1. J.D. Achenbach, Elastic waves and ultrasonic nondestructive evaluation, (1990)

2. J. Mandel, Rheology and Soil Mechanics, IUTAM, Springer-Verlag, (1964)

3. M. Emami Tabrizi, Stability Threshold of geomaterials ..., Ph.D. Thesis, Ecole Poly. X, (2008)

4. M. Emami Tabrizi, Luong M.P., Halphen, IRF’2009, (Eds: J.F. S-Gomes \& S.A. Meguid, 2009) 